\title{
Pankoke, Eckart
}

\section{"Offene Ganztagsschule" und "Kulturen der Verantwortung". Netzwerke und Lernprozesse schulischer Selbststeuerung}

Hansel, Toni [Hrsg.]: Ganztagsschule. Halbe Sache - großer Wurf? Schulpädagogische Betrachtung eines bildungspolitischen Investitionsprogramms. Herbolzheim : Centaurus 2005, S. 182-198. (Schulpädagogik; 7)

Quellenangabe/ Reference:

Pankoke, Eckart: "Offene Ganztagsschule" und "Kulturen der Verantwortung". Netzwerke und Lernprozesse schulischer Selbststeuerung - In: Hansel, Toni [Hrsg.]: Ganztagsschule. Halbe Sache großer Wurf? Schulpädagogische Betrachtung eines bildungspolitischen Investitionsprogramms. Herbolzheim : Centaurus 2005, S. 182-198 - URN: urn:nbn:de:0111-opus-34797 - DOI: 10.25656/01:3479

https://nbn-resolving.org/urn:nbn:de:0111-opus-34797 https://doi.org/10.25656/01:3479

in Kooperation mit / in cooperation with:

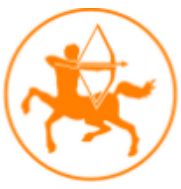

CENTAURUS

\section{Verlag \& Media KG}

\section{Nutzungsbedingungen}

Gewährt wird ein nicht exklusives, nicht übertragbares, persönliches und beschränktes Recht auf Nutzung dieses Dokuments. Dieses Dokument ist ausschließlich für den persönlichen, nicht-kommerziellen Gebrauch bestimmt. Die Nutzung stellt keine Übertragung des Eigentumsrechts an diesem Dokument dar und gilt vorbehaltlich der folgenden Einschränkungen: Auf sämtlichen Kopien dieses Dokuments müssen alle Urheberrechtshinweise und sonstigen Hinweise auf gesetzlichen Schutz beibehalten werden. Sie dürfen dieses Dokument nicht in irgendeiner Weise abändern, noch dürfen Sie dieses Dokument für öffentliche oder kommerzielle Zwecke vervielfältigen, öffentlich ausstellen, aufführen, vertreiben oder anderweitig nutzen.

Mit der Verwendung dieses Dokuments erkennen Sie die Nutzungsbedingungen an.

\section{Kontakt / Contact:}

\section{peDOCs}

DIPF | Leibniz-Institut für Bildungsforschung und Bildungsinformation Informationszentrum (IZ) Bildung

E-Mail: pedocs@dipf.de

Internet: www.pedocs.de

\section{Terms of use}

We grant a non-exclusive, non-transferable, individual and limited right to using this document.

This document is solely intended for your personal, non-commercial use. Use of this document does not include any transfer of property rights and it is conditional to the following limitations: All of the copies of this documents must retain all copyright information and other information regarding legal protection. You are not allowed to alter this document in any way, to copy it for public or commercial purposes, to exhibit the document in public, to perform, distribute or otherwise use the document in public.

By using this particular document, you accept the above-stated conditions of use. 


\section{GANZTAgSSCHULE. Halbe Sache - grober Wurf?}

Schulpädagogische Betrachtung eines bildungspolitischen Investitionsprogramms

Toni Hansel (Hg.)

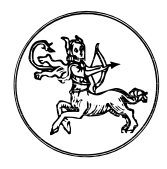

Centaurus Verlag

Herbolzheim 2005 
Der Herausgeber, Prof. Dr. Toni Hansel, ist Professor für Schulpädagogik an der Universität Rostock und Direktor des Instituts für Schulpädagogik.

Die Deutsche Bibliothek - CIP-Einheitsaufnahme

Bibliographische Information der Deutschen Bibliothek Die Deutsche Bibliothek verzeichnet diese Publikation in der Deutschen Nationalbibliographie; detaillierte bibliographische Daten sind im Internet über http://dnb.ddb.de abrufbar.

ISBN 3-8255-0614-2

\section{ISSN 1616-7414}

Alle Rechte, insbesondere das Recht der Vervielfältigung und Verbreitung sowie der Übersetzung, vorbehalten. Kein Teil des Werkes darf in irgendeiner Form (durch Fotokopie, Mikrofilm oder ein anderes Verfahren) ohne schriftliche Genehmigung des Verlages reproduziert oder unter Verwendung elektronischer Systeme verarbeitet, vervielfältigt oder verbreitet werden.

() CENTAURUS Verlags-GmbH \& Co. KG, Herbolzheim 2005

Umschlaggestaltung: Antje Walter, Hinterzarten

Satz: Vorlage des Herausgebers

Druck: primotec-printware, Herbolzheim 


\section{Disposition}

Grußwort des Dekans der Philosophischen Fakultät

Prof. Dr. Wolfgang Sucharowski

Schulpädagogische Betrachtung eines

bildungspolitischen Investitionsprogramms

$2.1 \quad$ Literatur

Eröffnung des Symposiums durch den Studiendekan der Philosophischen Fakultät

Prof. Dr. Wolfgang Nieke

Ganztagsschule als Bestandteil von Ganztagsbildung

\section{Perspektiven einer revitalisierten Reformidee}

Toni Hansel

Ganztagsschule - Angebot für eine Optimierung von Unterrichtsversorgung und Unterrichtsqualität

Ganztagsschule - nicht Erweiterung, sondern Vertiefung des

Schulzweckes

Voraussetzungen für die Einrichtung von Ganztagsschulen 
5.1 Reformpädagogik ohne Bedeutung für eine moderne Ganztagserziehung?

5.2 Beiträge der deutschen Reformpädagogik zu Konzeption und Realisierung moderner Formen der Ganztagsschule 39

$\begin{array}{lll}\text { 5.2.1 Die Bedeutung der deutschen Landerziehungsheime } & 39\end{array}$

5.2.2 Die Bedeutung der Wald- und Freiluftschulbewegung 43

5.2.3 Die Bedeutung der Arbeitsschulbewegung 48

5.2.4 Die Bedeutung der Schulpädagogik Herman Nohls 50

5.3 Reformpädagogisch gestaltete Ganztagsschule als Schule der Zukunft?

\section{Ganztagsschule und gesellschaftliche Vernetzung}

\section{Thomas Coelen}

Ganztagsbildung: Qualifikation und Partizipation von Kindern und Jugendlichen auf kommunaler Basis

6.1 Zur Idee hinter dem Begriff, Ganztagsbildung 57

6.2 Unterscheidung von Ganztagsschule, Ganztagsbetreuung und Ganztagsbildung

6.3 Anknüpfungen an gesellschaftstheoretisch gerahmte Bildungsbegriffe

Die Bedeutung der Ganztagsschule für den Wirtschaftsstandort Deutschland 


\title{
Ganztagsschule als bildungspolitische Option
}

\author{
Michael Becker/Ute Debold
}

Entwicklungsschub für Ganztagsschulen in

Mecklenburg-Vorpommern als landespolitische Aufgabe nach PISA

8.1

$\begin{array}{ll}\text { Einleitung } & 102\end{array}$

8.2

Die Ganztagsschule in gebundener Form

104

8.3

Das Evaluierungsprogramm Mecklenburg-Vorpommern

Probleme, Notwendigkeiten und Möglichkeiten dieser

Schulform

108

Ziele und Kriterien der pädagogischen Konzepte

Grundpositionen der Unterrichtsgestaltung an

Ganztagsschulen

Resümee

\section{Jörg-Dieter Gauger}

Ganztagsschule zwischen bildungspolitischer Opportunität und schulpädagogischer Konzeptlosigkeit

Zur Spannung zwischen Schulpädagogik und Bildungspolitik 


\title{
Neue Wege aus dem schulpraktischen Reformstau?
}

\author{
Angela Schulz
}

Ganztagsschulen und die richtige Antwort auf PISA: Ein Erfahrungsbericht

\section{Bernd Ostermeyer}

Ganztagsschule und Ganztagsbetreuung auf dem Prüfstand schulpraktischen Alltagshandelns

11.1 Jugend und Schule in veränderter Gesellschaft 152

11.2 Was kann und was soll Schule leisten? 154

11.3 Ganztagsschulen und Ganztagsbetreuung realistisch betrachtet 157

11.4 Zum Umgang mit dem 'Investitionsprogramm Zukunft, Bildung und Betreuung im schulischen Alltag'

\section{Eckhardt Preuß}

Leistungserziehung und Leistungsbeurteilung in der Grundschule/Schule im Blick auf die Ganztagsschule

12.1 Vorbemerkung zur Problematik und Inhalt des Themas

Die Welt der Kinder heute als Herausforderung für die Neugestaltung von Schule (Halbtags-Ganztagsschule) und Unterricht im Blick auf den Baustein „Leistungserziehung und Leistungsbeurteilung“

12.3 Pädagogisches Sehen und „pädagogisches Verstehen“ (W. Klafki ) - kritische Anmerkungen zu den alten und neuen Grundschulrichtlinien von NRW 1985 und 2003

12.3.1 Zentrale Aussagen der Richtlinien von 1985 anhand der „doppelten Reckstange“

12.3.2 Zentrale Aussagen der Richtlinien 2003 anhand einer „normierten“ Reckstange“

12.4 Fünf grundlegende Ziele der Leistungserziehung in Korrespondenz zu den fünf Tätigkeiten des Kindes 173 
12.6 Pädagogische Leistungsbeurteilung im Sinne und in der Ausgestaltung von Lernentwicklungsberichten 177

12.7 Literatur

\title{
Kulturelle Netzwerke und schulische Verantwortung
}

\author{
Eckart Pankoke
}

„Offene Ganztagsschule“ und „Kulturen der
Verantwortung“. Netzwerke und Lernprozesse
schulischer Selbststeuerung

Schul-Geschichte(n)

Neue Perspektiven und Horizonte

13.7 Selbständigkeit und Selbststeuerung der Organisations- und Relationsentwicklung

\section{Cristina Allemann-Ghionda}

Ganztagsschule internationalen Vergleich - von der Opposition zur Arbeitsteilung zwischen Staat und Familie? 
$\begin{array}{lll}\text { 14.3.3 } & \text { Erwerbstätige Mütter - akzeptiert } 209\end{array}$

14.3.4 Vorschulerziehung und Chancengleichheit 209

14.3.5 Täglicher Ablauf und staatliche Aufsicht 210

14.3.6 Kommentar 211

Pädagogische Argumente - kulturell gebunden oder
transnational?

14.4.1 Politische und soziale Argumente, psychologische und

14.4.2 Die Ganztagsschule in Italien 214

14.4.3 Ganztagsschule in der Schweiz 216

14.4.4 Ganztagsschule in Russland 217

$\begin{array}{lll}14.5 & \text { Schluss } & 219\end{array}$

14.6 Literatur 222

15 Toni Hansel

Was leistet Ganztagsschule? $\quad 224$

$15.1 \quad$ Vorbemerkung 224

15.2 Modellalternativen ganztagsschulischer Förderung 226

15.3 Welche Erwartungen verbinden sich mit der Einrichtung von Ganztagsschulen? 229

$15.4 \quad$ Wachsender Konsens 232

15.5 Was leistet die Ganztagsschule, was die Halbtagsschule nicht leisten kann? 235

15.6 Fazit 248

$\begin{array}{lll}15.7 & \text { Literatur } & 248\end{array}$

16 Autorenspiegel 250

$17 \quad$ Namensregister 


\section{3 „Offene Ganztagsschule“ und „Kulturen der Verantwortung“. Netzwerke und Lernprozesse schulischer Selbststeuerung.}

\subsection{Schul-Geschichte(n)}

Die alten Volks-Schulen pflegten ihre „pädagogische Provinz“ als „geschlossene Anstalt". Die Autorität der Lehrer gründete in einem festen Bildungskanon, den es zu vermitteln galt nach der Logik des „Trichters“. Als zentrales Organ schulischen Lernen galt das „Ohr“, - und mit dem Hören lernten die Schüler zugleich „Gehorsam“. „Wer nicht hören will, muss füblen!" - hieß es vielsagend. Gelernt wurde damit eher Disziplin als Reflexion. Lernmethode war eher das Diktat als der Diskurs. Und die Lehrer nannte man mit Recht „Pauker" und die Schule „Penne“. Ich selbst komme aus dem Ruhrgebiet und unsere Schule nannte man „Zeche“.

Aber die alten Volksschulen waren Vormittags-Schulen: die Nachmittage blieben frei für Schularbeiten, aber auch für hauswirtschaftliche oder auch landwirtschaftliche Mitarbeit. Informelle Spielwelten ergaben sich im häuslichen Umfeld. Ein Teil des freien Zeitfensters wurde besetzt durch die Angebote des örtlichen, in der Regel kirchlichen oder sportlichen Vereinslebens.

Einiges dieser alten Zeit hat sich immer noch gehalten. Doch predigen schon seit langem die Reformpädagogen ganz andere Kulturen des Lernens: „Nicht für die Schule, für das Leben lernen wir“. Und diese sollte umgesetzt werden durch „offenen Unterricht“ 
und durch „freies Lernen“. Aber auch diese „reform-pädagogische Offensive" entfaltete sich im Schonraum einer schulischen Eigenwelt, die sich abschirmte von den äußeren Zwängen und dem „Ernst des Lebens“der Gesellschaft.

Schule blieb so ein in sich ,geschlossenes System", das sich den Anregungen wie den Herausforderungen der gesellschaftlichen „Umwelt“ nur schwer öffnen wollte und konnte. Auch die Schulverwaltung blieb bürokratisch fixiert, d.h. Intentionen und Investitionen kommen von oben und nicht aus der Schule und ihrer Umgebung. Die Akteure, die in der Schule und mit der Schule leben, seien es Lehrer, Schüler, Eltern waren befangen in der Selbstgenügsamkeit ihrer Illusion kleiner und heiler Welten. Dabei könnten sie in der Schule und mit der Schule etwas verändern, wenn sie die eigenen Potentiale gemeinsam entwickeln und entfalten würden.

Längst führte der Wandel der Gesellschaft und ihrer Werte zu ,veränderter Kindheit" mit neuen Konsum- und Medienwelten. Auch darauf musste sich Schule neu einstellen. Zugleich aber muss Schule die nachwachsende Generation vorbereiten auf neue Verantwortungen in Familie, Beruf und Öffentlichkeit.

Will die Schule vorbereiten auch für das öffentliche Leben und die heute geforderten zivilgesellschaftlichen Kulturen der Verantwortung, gilt es, Brücken zu bauen zu außerschulischen Lernwelten und Verantwortungsfeldern. ${ }^{1}$,

Damit gewinnen Netzwerke und Lernprozesse - gerade im beruflichen wie politischen Praxisbezug moderner Bildungs-Einrichtungen - praktisches Interesse.

Schon 1844 forderte der Pädagoge Diesterweg von der modernen Schule, dass sie sich richten müsse ,gegen alles Hierarchische, Bevormundende und dergleichen", dass sie

1 Die über die Schulen zu vermittelnden Kulturen der Verantwortung reichen vom lokalen Engagement der Schulen in den kommunalen „Agenden21“ bis hin zu den auch im deutschen Bildungssystem aufgegriffenen neuen Horizonten der UN-Dekade „Bildung für eine nachhaltige Entwicklung“. 
,fraternisiert und sympathisiert, ... mit allem, was sich bewegt, sich entwickelt, fortbildet, lebt. Es ist das Prinzip des Fortschritts".2

\subsection{Neue Perspektiven und Horizonte}

Heute gewinnen solche Reformperspektiven neue Aktualität: „Selbständige Schule“, „offene Ganztagsschule“ und „lebensbegleitendes Lernen“ sind die neuen Themen. „Nicht in der Schule, im Leben lernen wir" - würde man dazu sagen. Das bedeutet praktisch, dass sich die Schule der Praxis von Wirtschaft und Gesellschaft interaktiv öffnen muss. Erlernt werden wollen nun neue „Kulturen der Verantwortung“ in den praktischen Feldern sozialen Lernens.

„Offene Ganætagsschule“ ${ }^{3}$ hatte zunächst eine zeitliche Dimension. Nicht nur die Schulstunden, der ganze Tageslauf der Schüler war nun pädagogisch zu verantworten. So kam der Alltag als pädagogisches Feld ,ganztägig“ in den Blick, weil in der alltäglichen Lebenssituation nicht nur für die einkommensschwachen und bildungsfernen Schichten, sondern auch in den sog. besser situierten Lagen kein produktives Lernklima mehr zu erwarten ist, - gerade für das soziale Lernen.

„Elternhaus und Schule“ - so hieß einmal eine weit verbreitete pädagogische Zeitschrift. Aber eine solche „Lernallianz“" ist heute nicht mehr vorauszusetzen, vielleicht

2 Adolph Diesterweg, Wegweiser zur Bildung für deutsche Lehrer (1834, 4. Aufl. 1844) Neudr. Paderborn 1958, 165. Vgl. Reinhart KoselLeK, "Fortschritt", in: Geschichtliche Grundbegriffe, Bd. 2, Stuttgart 1975, 398.

${ }^{3}$ Vgl. Thesen zum 4. Bildungsforum RuHr „Wir gehen aufs Ganze - Auf dem Weg zur offenen Ganztagsgrundschule“ (1.12.03):

1. Die Einführung der offenen Ganztagsgrundschule ist ein ambitioniertes Programm des Landes NRW, das einer integrierten Gestaltung zwischen unterschiedlichen Akteuren und Politikfeldern bedarf. (...)

2. Hierzu sollen möglichst viele Felder des gesellschaftlichen Umfelds wie bspw. Musikschulen, Malschulen, Sportvereine integriert werden. Da die Kooperationsbeziehungen zwischen Schulen und gesellschaftlichem Umfeld schwach ausgeprägt sind, gilt es zu überlegen, wie entsprechende Vernetzungsaktivitäten vor Ort aussehen können. 
auch nicht mehr ausreichend, weil die heimlichen Erzieher der aktuellen Erlebnisund Risikogesellschaft, gerade jenseits von Elternhaus und Schule zum Problem werden. Das hat zu tun mit dem Wandel der Familie, dem kulturellen Wandel der Werte, oder allgemein mit dem strukturellen Wandel der sozialen Welten.

Jenseits von Schulzeit und häuslichen Schularbeiten wird die sog. „Freizeit“allzu oft zum riskanten „Leerlauf“. Hier könnten die Angebote von „Ganztagsschule“ kompensatorisch gegensteuern. Zum anderen bedeutet das Projekt „Ganztagsschule“ den Anspruch, mit den Eltern neue Lern-Partnerschaften aufzubauen. Eltern sind in der Rolle von Co-Pädagogen gerade auch von der professionellen Pädagogik ernst zu nehmen.

Es gibt erste Bestätigungen, dass Eltern diese neue Partnerschaft mit der Schule aktiv bejahen, ${ }^{4}$ gerade auch in den additiven Angeboten. Das gilt nicht nur für von $70 \%$ der Eltern bejahte Hausaufgabenhilfe und die Hilfen zu aktiver Freizeitgestaltung. Neue Lern-Partnerschaften eröffnen sich dann auch über den Einsatz außerschulischer Fachkräfte aus unterschiedlichen Feldern der sozialen und kulturellen Praxis.

\subsection{Selbstgestaltung neuer Zeiten und Räume}

Aber „Ganztagsschule“ ist nicht nur eine neue Zeitstruktur, welche leer laufende Freizeit in Lernzeit verwandeln will. Es geht nicht nur um neue Zeiten, sondern auch um nene Räume. Pädagogisch formuliert: Es geht um die Aktivierung außerschulischer Lernfelder - und gelernt werden soll nicht nur auf der Ebene kognitiven Wissens und normativen Wertens, sondern auch in der Sinndimension sozialer Praxis.

\footnotetext{
${ }^{4}$ Vg. Polis: Die Ganztagsschule in Rheinland-Pfalz aus der Sicht der Eltern. - Ergebnisse einer Wiederholungsbefragung.
} 
Insbesondere ist die Ganztagsschule offen für neue Felder einer praktischen „Kultur der Verantwortung".

Praktisch bedeutet dies, dass nun neue Zeiten und Räume zu erschließen sind, in denen der traditionelle Rahmen einer „Schule als geschlossene Anstalt“ sich öffnen wird.

Die neuen „Zeit-Räume“ sind kulturell so zu gestalten, dass sie zur Identifikation und Integration einladen.

Im Ganztagsbetrieb braucht es Rückzugsräume für die Entspannung und Erholung. Es braucht Freiräume, die auch dem Bewegungsdrang junger Menschen gerecht werden. Die Räume müssen freundlich und hell sein und sollen organisch zur Schule dazu gehören und nicht ein Fremdkörper sein. Zu entwickeln sind aber auch kindgerechte Esskulturen und Speiseräume. Die richtige Ernährung spielt für das Lernen und das Wohlbefinden eine wichtige Rolle.

Immer geht es auch um „Lernallianzen“, in denen der Lehrer zurücktritt in die Rolle des Moderators und die Schüler ihre Lernprozesse selbst organisieren in der Begegnung mit offenen Feldern sozialer Praxis. Die könnte sich realisieren über die Begehung und Begegnung mit den unterschiedlichen Berufs- und Arbeitswelten. Ein anderes Feld wäre die Auseinandersetzung mit moderner Kunst. Zunehmend lebenswichtiger wird der Praxisbezug der Felder Sport, Gesundheit, Ernährung.

Kulturen der Verantwortung wären vor allem aber zu erkennen und zu erlernen in Lernallianz mit dem sog. „Dritten Sektor“ der Selbstorganisation freien Engagements, also kooperativ mit Jugendverbänden, Sportverbänden und sozialen Bewegungen für „Friede, Gerechtigkeit und Bewahrung der Schöpfung““ 


\subsection{Projekt-Partnerschaften}

Für die professionelle Steuerung dieses neuen Miteinanders sind Kooperationsverträge der Landesverbände der Lehrer, aber auch der Eltern notwendig. Die Einbindung neuer Kooperationspartner erfordert Verhandlungen mit der kommunalen Schulverwaltung und den entsprechenden Kulturministerien. Diese Verträge müssen den Einsatz von Fachkräften aus den jeweiligen Verbänden und Initiativen grundlegend regeln. Die Bedürfnisse und Bereitschaften der Kooperationspartner sind abzusprechen auf der Ebene von Schulorganisation und Schulkonferenz. Neue Verständigung gilt den pädagogischen Zielsetzungen. Dazu gehören alle Rechte und Pflichte beider Seiten.

\subsection{Außerschulische Kooperations-Partner}

Neue Partner außerschulischen Lernens wären dann auch die Unternehmer der Wirtschaft ${ }^{5}$, die Verantwortungsträger in Politik und Verwaltung, die Repräsentanten aus Kunst oder auch Kirche und die haupt- wie ehrenamtliche Aktivisten des „Dritten Sektors“. Im Sinne einer interkulturell und interreligiös „offenen Gesellschaft" wird auch die Begegnung mit den fremden Welten und Werten neuer Mitbürger zu einem zukunftswirksamen Lernfeld.

\footnotetext{
5 Vgl. 5. BiLdungSFORUM RuHR „Für das Leben lernen wir - Kooperation von Schule und Wirtschaft“ (Herten 26.2.2004): „Schüler/innen und Lehrer/innen erfahren durch die Kooperation mit den Betrieben konkret die Anforderungen der Arbeitswelt. Möglichkeiten der praktischen Zusammenarbeit helfen eigene Fähigkeiten besser einzuschätzen, tragen zur Transparenz von Berufen bei und erhöhen die Lernmotivation durch die Verknüpfung von Theorie und Praxis. $\langle\ldots\rangle$ Schulkooperationen mit der Wirtschaft zeichnen sich durch Vielfalt und Kreativität aller Beteiligten aus. $\langle\ldots\rangle$ Wichtige Voraussetzungen bestehen in der Öffnung der Schule gegenüber dem regionalen Umfeld. sowie in ausreichend bereitgestellten Ressourcen seitens der Schule und der Betriebe. Ein größeres Maß an Selbständigkeit und freien Handlungskompetenzen der Schulen unterstützt solche Kooperationsprozesse.“
} 
Doch die dazu geforderten Lernpartner müssen aber erst einmal gefunden und gewonnen werden. Das bedeutet Kosten, bringt aber auch produktive Kontexte des Vermittelns zwischen praktischer Erfahrung und schulischem Lernen. ${ }^{6}$

Die neuen Aufgaben der Ganztagsschule fordern deshalb auch ein kreatives Management der sich weitenden Ausgaben. Dies bedeutet das Einwerben von Drittmitteln und Zuwendungen. Auch Schulen müssen sich nun bemühen um Fundraising gerade auch im Bezug auf ein Corporate Citizenship der wirtschaftlichen Unternehmen. Dabei geht es nicht nur ums Geld, sondern auch um Lernpartnerschaften zur Vermittlung der Kompetenzen wirtschaftlicher Verantwortung, ${ }^{7}$ Ganztagsschule fordert also nicht nur die Entwicklung der schulischen Organisationsstrukturen; gefordert sind zugleich neue Relationen zum gesellschaftlichen Umfeld. Dabei geht es nicht nur um Geldmittel sondern auch um das „soziale Kapital“" partnerschaftlicher Kooperation. Zu denken ist an ehrenamtliches Engagement insbesondere aus der Elternschaft, an Corporate Volunteering, an die Zusammenarbeit mit den freien Vereinigungen des Dritten Sektors oder auch an die Aktivierung nach-beruflicher Kompetenz nach dem Modell Senior Consulting. Diese praktischen Kompetenzen des Organisierens und des Moderierens müssen an den Schulen erst erlernt werden. Zugleich muss sich an den Schulen eine neue Steuerungsfähigkeit entwickeln.

Hier ist gewiss das „Ehrenamt ${ }^{\star c 8}$ von Elternvertretung und Schülermitbestimmung mit in die Verantwortung gerufen. So bedeutet die „offene Ganztagsschule“ die Anerkennung neuer Herausforderungen für die Elternvertreter wie auch für die Schüler/innen in ihren Selbstverwaltungsämtern als Schul- und Klassensprecher.

6 Als Beispiel für außerschulische Lernfelder zum Thema „Umwelt“ vgl. das GELSENWASSERSchulprojekt, Zwischenbilanz 2004. Als Beispiel von „Corporate Citizenship“ bei der Förderung außerschulischer Felder kulturellen Lernens vgl. den „Kulturwettbewerb des Ruhrgebiets für Azubis, Schüler und Schulen“ (K.R.A.S.S.) der Unternehmen Ruhrkohle (RAG), WAZ und Karstadt-Quelle.

${ }^{7}$ Vgl. partnerfuerschule.nrw, Stiftung der Wirtschaft und des Schul- und Jugendministeriums NRW.

8 Oft engagieren sich Eltern ehrenamtlich etwa bei Schülerbücherei, Schulgartenpartnerschaft, Theater-AG, Gesundheits-Projekten. 
Dabei geht es nicht nur um Mitbestimmung in den inneren Angelegenheiten des Schullebens, sondern auch um die Mitgestaltung der Außenrelationen zwischen Schule und gesellschaftlichem Umfeld. Dies fordert Offenheit für eine professionell anspruchsvolle Kooperation mit dem Dritten Sektor (von der Schulsozialarbeit', über die Kunstpädagogik bis hin zu sportlichen Trainings-Programmen).

All dies aber ist nicht zum „Nulltarif“ zu haben, sondern wird auch für die kooperierenden Dritt-Sektor-Organisationen im eigenen sozialwirtschaftlichen Überlebenskampf zum wichtigen Geschäftsfeld. So ist die offene Ganztagsschule keine billige und willige Ergänzung zur Schulzeit, sondern erfordert eigene Ressourcen, die aus öffentlichen Mitteln oder auch freien Geldern von Stiftern und Sponsoren, aber auch durch sozialverträglich zu gestaltende Beiträge der Eltern aufzubringen sind.

Eine produktive Quelle der ehrenamtlichen Aktivierung nachberuflicher Kompetenz ergibt sich aus der Einbindung der berufspraktischen Kompetenzen und Kontexte aktiver Senioren. Das für Existenzgründer so hilfreiche Modell des „Senior-Consulting“ (auch „Senior-Coaching“) wäre auch auf die Schulen zu übertragen, indem Senioren ihre Praxiserfahrung einbringen in schulische Lernfelder. $\mathrm{Zu}$ verweisen ist auf die Kooperation mit ehemaligen Ausbildern der Sparten Holz, Metall und Bau in der von der ArAL-Stiftung (jetzt: Deutsche BP-STiftung) unterstützten Lehrwerkstatt der Hauptschule Essen-Karnap..$^{10}$ In einem ähnlichen Ansatz in der Stöver-Realschule Oer-Erkenschwick konnte das Projekt „Mädchen und Technik“ (MuT) unter der ehrenamtlichen Anleitung des Ausbildungspersonals

\footnotetext{
${ }^{9}$ Bei der Goethe-Schule in Herten wird die sozialarbeiterische und sozialpädagogische Begleitung durch Kooperationsvertrag mit der Arbeiterwohlfahrt geregelt. Die Betreuung erfolgt durch eine Erzieherin und Ergänzungskräfte, die bei der AWO angestellt ist. In der Essener Grundschule am Morungenweg wird die Ganztagsbetreuung in enger Kooperation mit der Schulpraxis durch den Sozialdienst katholischer Frauen geleistet. Daneben besteht eine enge Kooperation mit dem örtlichen Turnverein, der durch eine ABM-Kraft tätig wird. Auch die geplante Kooperation mit der Folkwang Musikschule erfordert eine Kostenregelung.

${ }^{10}$ Deutsche BP-STIFung: Jugendarbeitslosigkeit vorbeugen und überwinden. Förderbeispiele und Hintergrundinformationen.
} 
der Handwerksbetriebe die hier in ihrer beruflichen Planung oft desorientierten Mädchen für technische Handwerksberufe ermutigen.

Als ein wichtiger Partner auf gemeinsamen Wegen zu „Kulturen der Verantwortung“" sind die Eltern als „Erziehungspartner“ zu akzeptieren und zu aktivieren. ${ }^{11}$

\subsection{Entwicklungs-Strategien der Schulreform}

Hier müssen auch Qualifizierungsstrategien ansetzen. Eltern sind nicht per se die besseren Erzieher. Sie sollten in die Lage versetzt werden, angemessen am Schulablauf zu partizipieren. In vielen Bundesländern wurden dazu über den Landeselternrat entsprechende Modelle entwickelt und vermittelt. Diese sollten von den Schulen an interessierte Eltern weiter geleitet werden. Genauso sollten Schulen die Eltern in ihrer Bereitschaft zur Mitarbeit unterstützen. Viele Erwachsene erfahren erst als Eltern, was bürgerschaftliches Engagement möglich machen kann.

Eine „innere Reform“ des Bildungswesens ist heute dringlich gefordert, gerade in Regionen des wirtschaftlichen, gesellschaftlichen, kulturellen oder auch demographischen Umbruchs. Das Bewusstsein dieses Umbruchs sollte bildungspolitisch zum Aufbruch werden. So ist es schulische Praxis bereits in vielen Bundesländern oder

\footnotetext{
11 Vgl. 3. BILDUngSFORUM RuHR „Verantwortung für Kinder - Eltern und Pädagogen machen gemeinsame Sache“ (Essen 3.10.03):

„Eltern wie Pädagogen brauchen sich für ihre verantwortungsvolle Aufgabe gegenseitig. Wenn sich beide Seiten in ihren durchaus unterschiedlichen Rollen als Erziehungspartner verstehen, kann sich eine vertrauensvolle und aufgeschlossen Atmosphäre für eine konstruktive Zusammenarbeit entwickeln. Aus diesem gemeinsamen Verständnis heraus kann die Erziehungskompetenz von Eltern gestärkt werden $\langle\ldots\rangle$ etwa durch Projekte wie das FUN-Projekt der Arbeiterwohlfahrt oder Elterngesprächskreis vor und während der Grundschulzeit.“ $<\ldots>$ „Die Kommunen können im Erziehungsund Bildungsprozess von Kindern und Jugendlichen Moderatorenrollen übernehmen. Ein ,Bündnis für Erziehung' ist ein sinnvolles Instrument, um die notwendigen Kommunikations- und Interaktionsprozesse zwischen den Erziehungsverantwortlichen funktionsfähiger zu machen."
} 
auch im Ruhrgebiet. ${ }^{12}$ Dabei geht es weniger um Parolen als um konkrete Verfahren der Steuerung von Bildungsnetzen und Lernprozessen, etwa durch Wissensmanagement und prozess-orientiertes Qualitätsmanagement. ${ }^{13}$

\subsection{Selbständigkeit und Selbststeuerung der Organisations- und Relationsentwicklung}

Neue Aufgaben und neue Relationsmuster fordern neue Steuerung. Dabei gewinnen die Organisations- und Relationsprobleme der „offenen Ganztagsschule“ eine Komplexität, die den bürokratisch geschlossenen Rahmen herkömmlicher Schulverwaltung sprengen könnte. Auf die sich weitende Komplexität antwortet eine gesteigerte Reflexivität der Selbst-Steuerung. Reflexivität bedeutet praktisch den selbstkritischen Rückbezug auf die Bedürfnisse und Bereitschaften, die Erfahrungen und Empfindungen aller Beteiligten und Betroffenen. Dies gelingt über die Partizipation und die Selbstorganisation von Engagement und Interesse.

In einem historischen Exkurs können wir dazu weit ins 19. Jahrhundert rückblenden: In der „Verwaltungslehre“ des Lorenz VON STEIN war die sich weitende Komplexität der von ihm geforderten „Verwaltung der sozialen Reform“ nur zu meistern durch die Aktivierung gesellschaftlicher Selbstverwaltung. STEINs Würdigung der partnerschaftlichen Zusammenarbeit zwischen politischer Verwaltung und sozialem „Vereinswesen“ gilt als Weichenstellung zur modernen „Verwaltungslehre“ des „arbeitenden Staates“. Entsprechend müssen wir auch im Blick auf die Selbst-

\footnotetext{
12 Vgl. Bildungsoffensive Ruhrgebiet 2020. Nachhaltige strukturelle Veränderungen der Bildungssituation im Ruhrgebiet im Kontext des demografischen Wandels. Initiativpapier der Bürgermeister/innen und Oberbürgermeisterinnen der Kommunen.

${ }^{13}$ Vgl. ProjeKt Ruhr, Bildungsbeteiligung im Ruhrgebiet. Auf der Suche nach einer neuen Kompensatorik. Essen 2003:122. „Eine prozessorientierte Qualitätsentwicklung, die sowohl die Belange der Betroffenen und der einzelnen Organisation als auch zwischen den beteiligten Akteuren in den Blick nimmt, muss auf breiter Basis installiert werden,."
} 
verwaltung und Selbststeuerung der offenen Ganztagsschule die selbst-organisierten Interessen und Kompetenzen des gesellschaftlichen Umfeldes in den Blick nehmen.

Gerade die „Offene Ganztagsschule“ ist gefordert als „selbstständige Schule ${ }^{\text {“14 }}$. Die Doppelbindung dieser aktuellen schulpolitischen Programmformeln fassen wir steuerungstheoretisch als institutionelles Lernen von der „Selbstgenügsamkeit“ über die „Selbstregulierung“ zur „Selbststeuerung ${ }^{\text {(15 }}$.

„Selbstgenügsamkeit“ meinte eher jene traditionelle Schulkultur der autoritär „geschlossenen Anstalt“, welche im Sinne der alten Parole „Keine Experimente“ das Risiko von Innovationen eher scheut.

„Selbstregulierung“ hingegen steht für ein Reformklima, in dem sich die Schulen an die sich wandelnden Erwartungen von Wirtschaft und Gesellschaft thematisch und organisatorisch ,anpassen“, ohne damit jedoch das eingefahrene Verhältnis von Schule und Gesellschaft prinzipiell in Frage zu stellen.

„Selbststeuerung“ wird demgegenüber zur Programmformel dafür, dass nicht nur die Professionen und die Organisationen des Bildungsbereichs weiter entwickelt werden, sondern dass auch die Relationen des Bildungssystems zu seinen internen wie externen „Umwelten“ gesteuert wird über Projekt-Partnerschaften, wobei alle beteiligten Personen und Institutionen in die Steuerung einbezogen werden in partizipative „Kulturen der Verantwortung“.

\footnotetext{
14 Vgl. das gemeinsam von ProjeKt Ruhr GmbH, der Bertelsmann-STIFTung und BiLdungsFORUM RUHR durchgeführte Modellprojekt „Selbständige Schule“., Vgl. BILDUNGSFORUM RUHR: „Unternehmen ,Selbständigkeit"“(Bochum 14.10.2004).

15 Das strategische Konzept „Selbststeuerung“ ist übrigens erstmals entwickelt worden in einer Evaluation von Reformpolitiken der frühen 1970er Jahre als es galt, institutionelle Innovationen an den Beharrungstendenzen der etablierten Apparate vorbeizuspielen. Ein dafür besonders instruktives Beispiel war damals gerade die Bildungspolitik mit ihren Schulversuchen und Modellprojekten auf dem Wege zur Gesamtschule. Vgl. Eckart PANKOKE/ Hans NOKIELSKI/ Theodor Beine, Neue Wege gesellschaftlicher Selbststeuerung in der Bundesrepublik Deutschland. Diskussion an Beispielen aus den Bereichen Bildung, soziale Sicherung und kommunale Selbstverwaltung (Schriften der Kommission für wirtschaftlichen und sozialen Wandel 86), Göttingen 1975.
} 
Während bei „Selbstregulierung“ die Schule sich an neue Themen ,angepasst“ hat, bedeutet „Selbststeuerung“, dass die Schule selbst in ihrer Umwelt initiativ wird, indem sie das Lernen in außerschulische Lernfelder hinein verlegt. Das bedeutet eine sich weitende Komplexität und fordert eine gesteigerte Reflexivität der Steuerung von Bildungsprozessen. Selbststeuerung meint also eine strategische Steuerung des Wandels von Organisationen und deren Relationen in Selbst-Bestimmung und Selbst-Verantwortung aller Beteiligten und Betroffenen. ${ }^{16}$

Steuerungstheoretisch wechseln wir vom transitiven Top-Down-Diktat der „strategischer Autoritäten“ in die zunehmend reflexivere Bottom-Up-,Bewegung““ demokratischer „Agenden“.

In der Praxis der „,selbstständigen Schule“ stellen sich mit der Selbständigkeit neue, auch kritische Fragen:

„Wie baben nun die Schulen ibre neuen Handlungsspielräume ausgefüllt? Inwiefern hat die Organisations- und Personalentwicklung der Schulentwicklung genutzt? Besteht nicht auch die Gefahr, dass das Ziel des Projekts durch das Abschieben unliebsamer Verswaltungsarbeiten auf die Schulen bzw. die Schulleiter/innen verloren gebt und damit eher zu einer Verlagerung administrativer Aufgaben als zи Qualitätssteigerung fübrt? Sind Schulen nun auf dem Weg, Akteure regionaler Bildungspolitik zu werden, und entwickeln sich dabei neue Verantwortungspartnerschaften für Bildung und Erziehung in der Kommune? ‘d7

Antwort auf diese Frage geben die im „Bildungsforum Ruhr“ vorgestellten Reformprojekte bildungspolitischer Selbststeuerung, also die Freisetzung institutioneller Autonomie ,vor Ort“ - durch „Auskupplung“ aus den vertikalen

\footnotetext{
16 Selbststeuerung in der Sozialpolitik bedeutete damals die öffentliche Akzeptierung und Aktivierung „selbstaktiver Felder“ der selbst-organisierten Solidarität. Zur „Autonomie von Schule“ Vgl. Empfehlungen der Bildungskommission der Heinrich-Böll-Stifung: „Autonomie von Schule in der Wissensgesellschaft. Verantwortung in der Zivilgesellschaft, Berlin 2002.

${ }^{17}$ ProjekT Ruhr GmbH (Hg.) Unternehmen ,Selbständigkeit' - Schulen im Aufbruch. BILdungSFORUM RUHR 2004: 3.
} 
Kontrollmechanismen staatlicher Schulverwaltung und durch damit mögliche Freisetzung von Schulmitwirkung - gerade auch bei der Organisations- und Personalentwicklung. Diese neue Selbstständigkeit bedeutete gewiss auch neue Risiken, aber auch eine neue Offenheit für die Eigenverantwortung von Innovation:

Dies realisiert sich in der Gemeinschaftsgrundschule Bochum-Günnefeld in der praktizierten Vision eines „jahrgangsübergreifenden Unterrichts“, dessen Chance einer differenzierenden Förderung im Rahmen der Regelschule kaum möglich gewesen wäre.

- Einen stärkeren Einbezug der Erziehungskompetenz von Eltern mit der „Hammer Elternschule“, welche das Lernen lernbar machen sollte. Zugleich konnte in der Erziehungspartnerschaft mit den Eltern schulische und familiale Konflikte im Zusammenhang bearbeitet werden.

- Im Steinbart-Gynnasium Duisburg wurde das Konzept „das Lernen lernen“ praktisch, indem schulische Innovationen gemeinsam mit Lehrer/innen und Schüler/innen in einer professionell moderierten „Zukunftskonferenz“ entwickelt.

- In der Maria Sibylla Merian - Gesamtschule in Bochum wurden eingefahrene Sanktions- und Selektionsmechanismus wie das „Sitzen-Bleiben“ außer Kraft gesetzt. Gerade dies wird für die Lehrerschaft zur Aufforderung, sich mit den Problemen und den Potentialen gerade auch der schwächeren Schüler sensibler auseinanderzusetzen als zuvor. Zugleich versprach die Einführung neuer Modelle „Kooperativen Lernens“ ein „positiveres Lernklima, um die Kommunikations-. Sozial- und Teamkompetenzen aller Kinder und Jugendlichen in Gruppenarbeit zu fördern“.

- Dabei wird die Fremdkontrolle der vertikalen Schulaufsicht abgelöst durch neue Reflexionsformen der Selbstkontrolle (Team-Hospitation und Supervision). Gerade dann aber gehört eine auf die neuen Reformperspektiven fokussierte 
Weiterbildung zu den entscheidenden Garanten professioneller Qualitätssicherung.

\subsection{Offene Schule und aktive Öffentlichkeit}

Zwischen beiden bildungspolitischen Innovationen, dem neuen Modell „offener Ganztagsschule“ und den neuen Strategien „schulischer Selbststeuerung“ gibt es Wechselwirkungen. Die „offene Ganztagsschule“ entwickelt in ihren organisatorischen Schwierigkeiten, vor allem aber in ihren professionellen Möglichkeiten eine sich weitende Komplexität, die mit den herkömmlichen Mustern von Schulleitung und von Schulaufsicht nicht mehr zu steuern ist. Gefordert ist vielmehr eine neue Sensibilität und neue Reflexivität im verantwortlichen Umgang mit Problemen und Potentialen. Dies fordert eine neue politische Kultur der partizipativen Öffnung schulischer Selbststeuerung für die Mitwirkung von Lehrern, Eltern und vor allem auch der Schüler.

In dem Kooperationsprojekt der ProjeKT RuHR GmbH mit der Universität Essen „Lernallianzen im Ruhrgebiet - Bürgerschaftlichen Engagements“ galten als besondere Zielgruppe der Bürgergesellschaft die in der Schulmitwirkung als „Klassensprecher" engagierten Schülerinnen und Schüler. Auch hier wurde deutlich, dass ein schon früh gefordertes und entwickeltes Engagement sich langfristig nur akzeptiert und aktiviert sehen kann, wenn es auch in den Lernprozessen der Entwicklung partizipativer Kompetenzen ernst genommen wird. Die dazu im Rahmen des Projektes „Lernallianzen“ entwickelten Module und Workshops zur aktivierenden Qualifizierung der Schul- und Klassensprecher als Aktivkosten der Bürgergesellschaft 
wurde nicht nur von den Schüler/innen dankbar angenommen, sondern auch bei der Projekt-Evaluation als besonders zukunftswirksam gewürdigt. ${ }^{18}$

\section{9 (Inter-)kulturelle Netzwerke und Lernprozesse}

Ein Zukunftsthema ist auch der Beitrag der „offenen Ganztagsschule“ zum Begegnen der Kulturen: Zukunftweisend für das Zusammenwachsen zur offenen Gesellschaft wird das kulturelle und politische Klima an unseren Schulen und Hochschulen. Im Sinne einer interkulturellen „Kultur der Verantwortung“ muss es gelingen, auch die Mitschüler mit Migrationshintergrund - etwa über ihre „Berufung“ ins Amt des Klassensprechers - in ihren Verantwortungsrollen zu stützen und zu stärken. In den Schülerinnen und Schüler, die sich als Schul- und Klassensprecher für andere und ihre kleine Gemeinschaft engagieren, erkennen wir Zukunftspotentiale der Bürgergesellschaft. Hier liegt auch ein partizipatives Potential für die Selbststeuerung der Netzwerke und Lernprozesse der „offenen Ganztagsschule“. In deren Umfeld-Relationen entwickelt sich eine frühe 'Kultur der Verantwortung', der es dann bald nicht mehr nur um die Klassengemeinschaft geht und deren Vertretung gegenüber der Institution Schule. Oft sind es gerade die Klassensprecher, welche die Brücke schlagen zur Bürgergesellschaft, - etwa wenn aktive Schüler sich zugleich in den Vereinen und Initiativen des lokalen Umfeldes engagieren. So könnte eine von der Schule ausgehende Selbstorganisation auch wirksam werden für die Netzwerke und Lernprozesse der neuen Öffentlichkeit einer interkulturell interaktiven Gesellschaft.

\footnotetext{
${ }^{18}$ Vgl. Reinhild HugENROTH, „Schule und bürgerschaftliches Engagement - Lernallianzen in Nordrhein-Westfalen und Rheinland-Pfalz. Eine Untersuchung von Programmen und Initiativprojekten.“ (Arbeitsteil Dissertationsprojekt Universität Duisburg-Essen)
} 


\subsection{Bilanz und Perspektive}

Als „kleine und heile Welt“ war die alte Volks-Schule eine zentrale Institution der „machtgeschützten Innerlichkeit“ (so Thomas MANN) des „Deutschen Kulturstaats ${ }^{\text {(119. }}$. Ihre Struktur als geschlossene Anstalt war recht einfach gebaut im Zusammenspiel zwischen der pädagogischen Autorität der Lehrer/innen und der bürokratischen Autorität staatlicher Schulverwaltung.

Demgegenüber ist die „offene Ganztagsschule“ gefordert, sich interaktiv einzulassen auf die Praxis des beruflichen, kulturellen und öffentlichen Lebens mit den hier ungleich komplexeren Netzwerken und ungleich turbulenteren Lernprozessen. Das gilt für die Mikropolitik innerschulischer Spannungsfelder und für die sich hier vermittelnde schulische Mitbestimmung von Eltern und Schülern. Komplexität steigert sich aber auch in den außerschulischen Praxis-Partnerschaften und Kooperationsfeldern. Das alles lässt sich nicht von oben regulieren oder gar diktieren. Gefordert ist vielmehr eine partnerschaftliche „Kultur der Verantwortung“, die jeweils „,vor Ort“ aktiv werden muss. Die alten „Autoritäten“ der traditionellen Schule und ihrer Lehrer/innen ist somit zu überführen in eine neue „Autonomie“ schulischer Selbstständigkeit und Selbststeuerung, in welche alle Beteiligten partizipativ und interaktiv einzubeziehen sind.

„Schulen werden selbständiger." Dies wird deutlich an „Autonomisierungsprozessen in den Feldern der Personalwirtschaft sowie der Budgetierung von Geld- und Zeitressourcen. Deutscblands Bildungspolitik ist auf dem Weg dabin, den Schulen mebr Gestaltungsfreiräume ein₹uräumen." Aber: „Die selbständige Schule stellt Anforderungen, denen sich die Lebrerinnen und Lebrer genan so wie die Schulleitungen stellen mussten, auf die beiden Gruppen bisher auch wenig oder gar nicht vorbereitet wurden. $\langle\ldots\rangle$ Wie lange es dauern wird, bis aus dem Autonomisierungsprozess der

\footnotetext{
${ }^{19}$ E. PankoKe und K. Rohe, Der Deutsche Kulturstaat, in: Th. Ellwein / E. Holtmann (Hrsg.), 50 Jahre Bundesrepublik Deutschland. (Politische Vierteljahresschrift Sonderband 30), Opladen / Wiesbaden 1999, S. 168-180.
} 
Schulen eine neue Balance zwischen Scbulen, Schulaufsicht, und Scbulträgern erwächst, darïber lässt sich derzeit nicht einmal begründet spekulieren. Aber immerbin ein neuer Anfang ist gemacht. “20

Einen neuen Anfang fordert gerade die Ganztagsschule mit ihren komplexen Netzwerken und Lernprozessen, was sich nicht nur auf die „innere Führung“ einer modernen Schule beziehen, sondern was gerade auch in den Außenrelationen der unterschiedlichen Projektpartnerschaften eine neue (Selbst-)Steuerung entwickeln muss. Das gilt für ein neues Verhältnis von „Elternhaus und Schule“, es gilt für neue Kontakte zu den Praxisfeldern der Berufs- und Arbeitswelt und vor allem für ein neues partizipatives Engagement von Lehrern und Schülern „vor Ort“, in der aktiven Öffentlichkeit von zivilem Vereinswesen und kommunaler Selbstverwaltung.

${ }^{20}$ Klaus KLEMM/ Frank MEETZ, Schulen werden selbstständiger. In: EsSENER UNIKATE. Berichte aus Forschung und Lehre: Bildungsforschung nach PISA. Essen 2004, 16. 